\title{
Inclusão de celulose em rações para juvenis de tambacu
}

\author{
Inclusion of cellulose in diets for juvenile tambacu
}

\author{
BRAGA, Luís Gustavo Tavares ${ }^{1 *}$; AZEVEDO, Rafael Vieira de ${ }^{2}$; CIPRIANO, Filipe \\ dos Santos $^{3}$; LIMA, Kauana Santos ${ }^{4}$; MAGALHÃES JÚNIOR, Francisco Oliveira de ${ }^{1}$; \\ TONINI, William Cristiane Telles ${ }^{5}$; SANTOS, Danielle Ferreira dos ${ }^{1}$
}

\footnotetext{
${ }^{1}$ Universidade Estadual de Santa Cruz, Departamento de Ciências Agrárias e Ambientais, Ilhéus, Bahia, Brasil. ${ }^{2}$ Universidade Estadual do Norte Fluminense Darcy Ribeiro, Laboratório de Zootecnia e Nutrição Animal, Campos dos Goytacazes, Rio de Janeiro, Brasil.

${ }^{3}$ Universidade Federal de Minas Gerais, Minas Gerais, Minas Gerais, Brasil.

${ }^{4}$ Universidade Federal da Bahia, Escola de Medicina Veterinária e Zootecnia, Salvador, Bahia, Brasil.

${ }^{5}$ Universidade do Estado da Bahia, Xique-Xique, Bahia, Brasil.

*Endereço para correspondência: lgtbraga@gmail.com
}

\section{RESUMO}

Objetivou-se avaliar o desempenho zootécnico e a composição corporal de juvenis de tambacu alimentados com rações contendo níveis crescentes de celulose. 128 juvenis de tambacu $(29,0 \pm 6,0 \mathrm{~g})$ foram distribuídos em 16 tanques (150 L), na densidade de oito exemplares por tanque, em um delineamento inteiramente casualizado com quatro tratamentos (níveis crescentes de celulose: $0 ; 20 ; 40$ e $60 \mathrm{~g} \mathrm{~kg}^{-1}$ ) e quatro repetições. Os parâmetros de desempenho avaliados foram: peso final $(\mathrm{PF})$, consumo de ração (CR), ganho de peso (GP), conversão alimentar aparente (CAA), taxa de eficiência proteica (TEP) e sobrevivência (SOB). Para a análise de composição corporal, foram analisados matéria seca (MS), proteína bruta (PB), extrato etéreo (EE), energia bruta (EB) e matéria mineral (MM). Verificou-se comportamento quadrático $(\mathrm{P}<0,05)$ para as variáveis $\mathrm{PF}, \mathrm{CR}$, GP e CAA com os melhores resultados obtidos, respectivamente, para os níveis de 19,$95 ; 11,22 ; 19,87$ e $23,86 \mathrm{~g} \mathrm{~kg}^{-1}$ de inclusão da celulose nas rações. Não houve efeito $(\mathrm{P}>0,05)$ dos níveis de inclusão da celulose sobre a TEP, SOB e sobre os parâmetros de composição corporal MS, PB, EE, EB e MM. As rações com maiores níveis de inclusão da celulose afetaram negativamente o desempenho de juvenis de tambacu, no entanto, as modificações fisiológicas e metabólicas provocadas por essas rações não foram suficientes para afetar a composição corporal.

Palavras-chave: Colossoma macropomum, composição corporal, desempenho zootécnico, fibra alimentar, Piaractus mesopotamicus

\section{SUMMARY}

Aimed to evaluate the growth performance and body composition of juvenile tambacu fed diets containing increasing levels of cellulose. 128 juveniles $(29.0 \pm 6.0 \mathrm{~g})$ were distributed into 16 tanks $(150 \mathrm{~L})$, at a density of eight fish per tank, in a completely randomized design with four treatments (increasing levels of cellulose: 0, 20, 40 and $60 \mathrm{~g} \mathrm{~kg}^{-1}$ ) and four replications. For the growth performance parameters it was evaluated: final weight (FW), feed intake (FI), weight gain (WG), feed conversion rate (FCR), protein efficiency rate (PER) and survival (SUR). For the analysis of body composition, dry matter (DM), crude protein $(\mathrm{CP})$, ether extract (EE), gross energy (GE) and mineral matter (MM) were analyzed. There was a quadratic response $(\mathrm{P}<0.05)$ to the $\mathrm{FW}, \mathrm{FI}, \mathrm{WG}$ and FCR variables with the best results obtained, respectively, to levels of 19.95, 11.22, 19.87 and $23.86 \mathrm{~g} \mathrm{~kg}^{-1}$ of inclusion of cellulose in the rations. There was no effect $(\mathrm{P}>0.05)$ of levels of inclusion of cellulose on the PER, SUR and on the parameters of body composition in DM, CP, EE, GE and MM. Diets with high inclusion levels of cellulose adversely affected the performance of juvenile tambacu. However, the physiological and metabolic changes caused by these rations were not sufficient to affect body composition.

Keywords: body composition, Colossoma macropomum, dietary fiber, growth performance, Piaractus mesopotamicus 


\section{INTRODUÇ̃̃̃O}

A avaliação do uso de fibra alimentar na dieta de peixes tornou-se necessária devido à necessidade da utilização de fontes vegetais em substituição à farinha e óleo de peixe. Além disso, é sabido que o balanceamento correto das dietas e o conhecimento do nível de fibra alimentar modificam o funcionamento do sistema digestório e afetam $\mathrm{o}$ desempenho dos peixes (FABREGATT et al., 2011; LANNA et al., 2004a; RODRIGUES et al., 2010a).

Os efeitos fisiológicos da fibra dietética na digestão do alimento e na absorção de nutrientes são altamente influenciados pela sua natureza química e estrutura física (KROGDAHL et al., 2005). A fibra alimentar solúvel (pectinas, gomas e mucilagens) atua como componente ativo na regulação da digestão e absorção intestinal, e a insolúvel (celulose, lignina e hemicelulose) atua na formação do bolo alimentar, diluindo os nutrientes e diminuindo o tempo de trânsito gastrintestinal (CUMMING et al., 2004; KROGDAHL et al., 2005).

Os estudos com fibra alimentar para peixes apresentam grandes variações nos resultados. Rodrigues et al. (2012) avaliaram, para tilápia do nilo (Oreochromis niloticus) e jundiá (Rhamdia quelen), a inclusão de fontes vegetais com diferentes níveis de fibra alimentar. Os autores observaram que houve redução na digestibilidade da proteína, energia e matéria seca com o aumento de fibra dos ingredientes para ambas as espécies. No entanto, o desempenho zootécnico da tilápia correspondeu à digestibilidade das fontes vegetais, não se observando o mesmo padrão para os jundiás.

Geralmente, as diferenças nos resultados em estudos com fibra alimentar devem-se à grande diversidade entre as espécies estudadas, à complexidade, solubilidade e nível de inclusão de fibra nas dietas (LEENHOUWERS et al., 2007).

O tambacu é um híbrido obtido pelo cruzamento entre o macho pacu (Piaractus mesopotamicus) e a fêmea tambaqui (Colossoma macropomum). Apresenta hábito alimentar onívoro e reúne a rusticidade e a resistência a baixas temperaturas do pacu e a precocidade do tambaqui. Ao contrário do pacu e do tambaqui, poucas informações estão disponíveis sobre as exigências nutricionais do tambacu (PEREIRA et al., 2011).

Diante do exposto, objetivou-se verificar o desempenho zootécnico e a composição química corporal de juvenis de tambacu alimentados com rações contendo níveis crescentes de celulose.

\section{MATERIAL E MÉTODOS}

O experimento foi realizado no Laboratório de Nutrição e Alimentação de Peixes (Aquanut) da UESC, Ilhéus, Bahia, durante 60 dias. 128 juvenis de tambacu, adquiridos na Comissão Executiva do Plano da Lavoura Cacaueira (Ceplac) de Ilhéus, com peso inicial de 29,0 $\pm 6,0 \mathrm{~g}$, foram distribuídos em 16 tanques circulares de fibra de vidro, com volume útil de $150 \mathrm{~L}$, na densidade de oito exemplares por tanque. Os peixes passaram por um período de adaptação de 20 dias no Aquanut, alimentados três vezes ao dia com ração comercial extrusada com $320,0 \mathrm{~g} \mathrm{~kg}^{-1}$ de proteína bruta.

O abastecimento de água foi feito através de um sistema de circulação fechada, com uso do filtro biológico, com vazão de $1,4 \mathrm{~L} \mathrm{~min}^{-1}$, através do uso de bomba d'água (Dancor ${ }^{\circledR}$, RJ, 
Brazil - 3/4 cv). Cada tanque possuía aeração individual, por meio de pedra porosa, que era alimentada por um soprador (Mod. CV-101R, VENTBRAS Ind. Eletrometalúrgica Ltda, SP, BR - 1 $\mathrm{cv})$.

Ao longo de todo o período experimental, o monitoramento da qualidade da água foi feito com uso de equipamentos multiparâmetros (YSY modelo 63-10FT e YSY modelo 5512FT, YSI Corporation, Owings Mills, MA, USA) com os quais foram coletados dados de temperatura, oxigênio dissolvido, condutividade e $\mathrm{pH}$, cujos valores médios foram $27,5 \pm 1,2^{\circ} \mathrm{C} ; \quad 5,08 \pm 1,0 \quad \mathrm{mg} \quad \mathrm{L}^{-1}$; $277,1 \pm 50,3 \mu \mathrm{S} \mathrm{cm} \mathrm{cm}^{-1}$ e $7,6 \pm 0,3$. Para a retirada dos resíduos decantados no fundo dos tanques foi feita a sifonagem duas vezes por semana.

$\mathrm{O}$ experimento foi planejado em um delineamento inteiramente casualizado com quatro tratamentos e quatro repetições. Os tratamentos consistiam em dietas isoenergéticas (2890 $\left.\mathrm{kcal} \mathrm{kg}^{1}\right)$ e isonitrogenadas $\left(271,0 \mathrm{~g} \mathrm{~kg}^{-1}\right) \mathrm{com}$ níveis crescentes de celulose, a saber: 0 ; 20; 40 e $60 \mathrm{~g} \mathrm{~kg}^{-1}$, utilizando-se a celulose microfina Rhoster (Rhoster Indústria e Comércio Ltda, Vargem Grande Paulista, SP, Brasil) com 98\% de pureza e $78 \%$ de fibra bruta.

As formulações das dietas experimentais (Tabela 1) foram feitas com o auxílio do programa computacional SUPERCRAC ${ }^{\circledR}$ baseadas nas exigências nutricionais do pacu (ABIMORAD \& CARNEIRO, 2004; RODRIGUES et al., 2010b; WATANABE et al., 2010).

Para a confecção das rações experimentais, todos os ingredientes foram misturados em misturador industrial, umedecidos com $20 \%$ de água na temperatura de $40^{\circ} \mathrm{C}$ e em seguida peletizados em peletizadora com reversor (matriz de 2,5 $\mathrm{mm}$ ) e, posteriormente, desidratadas em estufa de ventilação forçada a $55^{\circ} \mathrm{C}$ por 24 horas e armazenadas em refrigerador durante todo o período experimental.

Durante o experimento, os peixes foram alimentados quatro vezes ao dia até a saciedade aparente. No início e ao final do experimento, foi realizada a pesagem total dos peixes de cada unidade experimental. Os parâmetros de desempenho produtivo avaliados foram: consumo de ração (consumo de ração/período experimental), ganho de peso [(peso final - peso inicial) / período experimental)], conversão alimentar aparente (consumo de ração / ganho de peso), taxa de eficiência proteica (ganho de peso consumo de proteína bruta) e sobrevivência [(indivíduos mortos / indivíduos vivos) $\mathrm{x}$ 100].

Para a análise de composição corporal, dois peixes por repetição, ao final do período experimental, foram eutanaziados com superdosagem do anestésico benzocaína $\left(120 \mathrm{mg} \mathrm{L}^{-1}\right)$. A seguir, os peixes foram moídos inteiros e secos em estufa a $65^{\circ} \mathrm{C}$ por 72 horas para análises de matéria seca, proteína bruta, extrato etéreo e matéria mineral, conforme metodologia descrita por Silva \& Queiroz (2002) e energia bruta através de bomba calorimétrica IKA C200 (IKA ${ }^{\circledR}$ - Werke Gmbh \& Co. KG, Staufen, Germany).

Os dados de desempenho e composição corporal foram submetidos à análise de variância a 5\% de probabilidade e, em caso de diferença estatística, aplicou-se a análise de regressão, utilizando o software R Core Team (2011). 
Rev. Bras. Saúde Prod. Anim., Salvador, v.15, n.4, p.947-956 out./dez., 2014 http://www.rbspa.ufba.br ISSN 15199940

Tabela 1. Composição $\left(\mathrm{g} \mathrm{kg}^{-1}\right)$ das dietas experimentais com a inclusão de celulose

\begin{tabular}{|c|c|c|c|c|}
\hline \multirow{2}{*}{ Ingrediente $\left(\mathrm{g} \mathrm{kg}^{-1}\right)$} & \multicolumn{4}{|c|}{ Nível de celulose $\left(\mathrm{g} \mathrm{kg}^{-1}\right)$} \\
\hline & 0 & 20 & 40 & 60 \\
\hline Farelo de soja & 400,0 & 395,8 & 365,4 & 361,8 \\
\hline Farelo de trigo & 189,1 & 50,0 & 50,0 & 50,0 \\
\hline Farelo de milho & 50,0 & 123,0 & 163,6 & 152,3 \\
\hline Amido de milho & 120,0 & 120,0 & 66,0 & 60,0 \\
\hline Farinha de carne e ossos & 160,0 & 134,9 & 160,0 & 160,0 \\
\hline Farinha de peixe & 70,7 & 140,0 & 140,0 & 140,0 \\
\hline Óleo de soja & 0,0 & 6,0 & 4,7 & 5,7 \\
\hline Celulose em pó & 0,0 & 20,0 & 40,0 & 60,0 \\
\hline Premix mineral e vitamínico ${ }^{1}$ & 10,0 & 10,0 & 10,0 & 10,0 \\
\hline Butilhidroxitolueno & 0,2 & 0,2 & 0,2 & 0,2 \\
\hline Parâmetro & \multicolumn{4}{|c|}{ Valores calculados $\left(\mathrm{g} \mathrm{kg}^{-1}\right)$} \\
\hline Proteína digestível & 269,0 & 271,0 & 271,0 & 269,0 \\
\hline Energia digestível ( $\left.\mathrm{kcal} \mathrm{kg}^{-1}\right)$ & 2890 & 2890 & 2890 & 2830 \\
\hline Gordura & 68,5 & 68,5 & 68,5 & 68,5 \\
\hline Fibra bruta & 40,02 & 43,46 & 58,15 & 73,36 \\
\hline Fibra em detergente neutro & 322,0 & 346,0 & 389,0 & 393,0 \\
\hline Fibra em detergente ácido & 32,0 & 46,0 & 74,0 & 124,0 \\
\hline Aminoácidos & \multicolumn{4}{|c|}{ Valores calculados $\left(\mathrm{g} \mathrm{kg}^{-1}\right)$} \\
\hline Lisina & 20,76 & 22,00 & 21,79 & 21,66 \\
\hline Metionina & 4,16 & 4,81 & 4,82 & 4,79 \\
\hline Metionina + Cistina & 7,89 & 8,63 & 8,61 & 8,55 \\
\hline Triptofano & 2,99 & 2,86 & 2,76 & 2,73 \\
\hline Treonina & 11,13 & 11,79 & 11,69 & 11,60 \\
\hline Arginina & 23,02 & 23,38 & 23,32 & 23,16 \\
\hline Valina & 15,23 & 16,03 & 15,94 & 15,82 \\
\hline Isoleucina & 13,16 & 13,78 & 13,49 & 13,38 \\
\hline Leucina & 22,85 & 24,36 & 24,17 & 23,94 \\
\hline Histidina & 7,37 & 7,58 & 7,48 & 7,41 \\
\hline Fenilalanina & 14,89 & 15,27 & 15,23 & 15,10 \\
\hline Fenilalanina + Tirosina & 22,43 & 23,31 & 23,15 & 22,95 \\
\hline
\end{tabular}

'Composição do premix mineral e vitamínico $\left(\mathrm{kg}^{-1}\right)$ : vit. $\mathrm{A}=6.000 .000 \mathrm{UI}$; vit. D3 = 2.250.000 UI; vit. $\mathrm{E}=75.000 \mathrm{mg}$; vit. $\mathrm{K} 3=3.000 \mathrm{mg}$; vit. tiamina $=5.000 \mathrm{mg}$; riboflavina $=10.000 \mathrm{mg}$; vit.pirodoxina $=$ $8.000 \mathrm{mg}$; biotina $=2.000 \mathrm{mg}$; vit. $\mathrm{C}=192.500 \mathrm{mg}$; niacina $=30.000 \mathrm{mg}$; ácido fólico $=3.000 \mathrm{mg}$; $\mathrm{Fe}=100.000 \mathrm{mg} ; \mathrm{Cu}=600 \mathrm{mg} ; \mathrm{Mn}=60.000 \mathrm{mg} ; \mathrm{Zn}=150.000 \mathrm{mg} ; \mathrm{I}=4.500 \mathrm{mg} ; \mathrm{Cu}=15.000 \mathrm{mg}$; $\mathrm{Co}=2.000 \mathrm{mg} ; \mathrm{Se}=400 \mathrm{mg}$.

\section{RESULTADOS E DISCUSSÃO}

Foi observada influência significativa do nível de inclusão da celulose sobre as variáveis peso final, consumo de ração, ganho de peso e conversão alimentar aparente (Tabela 2).

Verificou-se comportamento quadrático $(\mathrm{P}<0,05)$ para as variáveis de peso final, consumo de ração, ganho de peso e conversão alimentar aparente com os melhores resultados obtidos, respectivamente, para os níveis de 19,$95 ; 11,22 ; 19,87$ e $23,86 \mathrm{~g} \mathrm{~kg}^{-1}$ de inclusão da celulose nas rações (Figuras $1,2,3$ e 4$)$.

Não houve efeito $(\mathrm{P}>0,05)$ dos níveis de inclusão da celulose sobre a taxa de 
Rev. Bras. Saúde Prod. Anim., Salvador, v.15, n.4, p.947-956 out./dez., 2014 http://www.rbspa.ufba.br ISSN 15199940

eficiência proteica e sobrevivência (Tabela 2).

Corroborando com os resultados do presente estudo, Rodrigues et al. (2010b) trabalhando com níveis crescentes $(50 ; 70 ; 90 ; 110 ; 130$ e $150 \mathrm{~g}$ $\mathrm{kg}^{-1}$ ) de fibra bruta em rações para $\mathrm{o}$ pacu $(23,97 \pm 0,59 \mathrm{~g})$ observaram queda no ganho de peso e no consumo de ração com níveis acima de $110 \mathrm{~g} \mathrm{~kg}^{-1}$ de fibra bruta na ração. Pedron et al.
(2008), avaliando diferentes fontes (casca de soja ou de algodão) e níveis $\left(50,0 ; 73,0\right.$ e $\left.92,0 \mathrm{~g} \mathrm{~kg}^{1}\right)$ de fibra bruta para jundiás $(129,11 \pm 7,81 \mathrm{~g})$, não observaram diferenças em relação à sobrevivência, porém, observaram que os peixes alimentados com rações contendo $73,0 \mathrm{~g} \mathrm{~kg}^{-1}$, independente da fonte, apresentaram a maior biomassa final.

Tabela 2. Valores médios de desempenho zootécnico de juvenis de tambacu submetidos a diferentes níveis de celulose nas rações

\begin{tabular}{|c|c|c|c|c|c|c|}
\hline \multirow{2}{*}{ Variável } & \multicolumn{4}{|c|}{ Nível de celulose $\left(\mathrm{g} \mathrm{kg}^{-1}\right)$} & \multirow{2}{*}{ Valor de F } & \multirow{2}{*}{$\begin{array}{l}\text { CV } \\
(\%)\end{array}$} \\
\hline & 0 & 20 & 40 & 60 & & \\
\hline Peso inicial (g) & 26,90 & 26,90 & 26,90 & 26,90 & - & - \\
\hline Peso final $(\mathrm{g})^{1}$ & 70,92 & 82,59 & 68,41 & 52,63 & 13,47 & 11,61 \\
\hline Consumo de ração $\left(\mathrm{g} \mathrm{dia}^{-1}\right)^{2}$ & 1,15 & 1,21 & 1,01 & 0,80 & 5,93 & 17,35 \\
\hline Ganho de peso $\left(\mathrm{g} \mathrm{dia}^{-1}\right)^{3}$ & 0,73 & 0,93 & 0,69 & 0,43 & 13,47 & 11,61 \\
\hline Conversão alimentar aparente ${ }^{4}$ & 1,65 & 1,45 & 1,52 & 1,95 & 5,43 & 13,98 \\
\hline Taxa de eficiência proteica (\%) & 2,30 & 2,76 & 2,51 & 2,02 & 1,63 & 23,99 \\
\hline Sobrevivência (\%) & 80,57 & 80,57 & 80,57 & 88,90 & 2,78 & 9,25 \\
\hline
\end{tabular}

${ }^{1}$ Efeito quadrático $(\mathrm{P}=0,0007) \mathrm{Y}=-0,01716 \mathrm{x}^{2}+0,68463 \mathrm{x}+72,12118 ; \mathrm{R}^{2}=0,9354$.

${ }^{2}$ Efeito quadrático $(\mathrm{P}=0,0148) \mathrm{Y}=-0,00016717 \mathrm{x}^{2}+0,00375 \mathrm{x}+1,16220 ; \mathrm{R}^{2}=0,9684$.

${ }^{3}$ Efeito quadrático $(\mathrm{P}=0,0007) \mathrm{Y}=-0,00028508 \mathrm{x}^{2}+0,01133 \mathrm{x}+0,75502 ; \mathrm{R}^{2}=0,9360$.

${ }^{4}$ Efeito quadrático $(P=0,0200) Y=0,00038989 x^{2}-0,01861 x+1,65446 ; R^{2}=0,9962$.

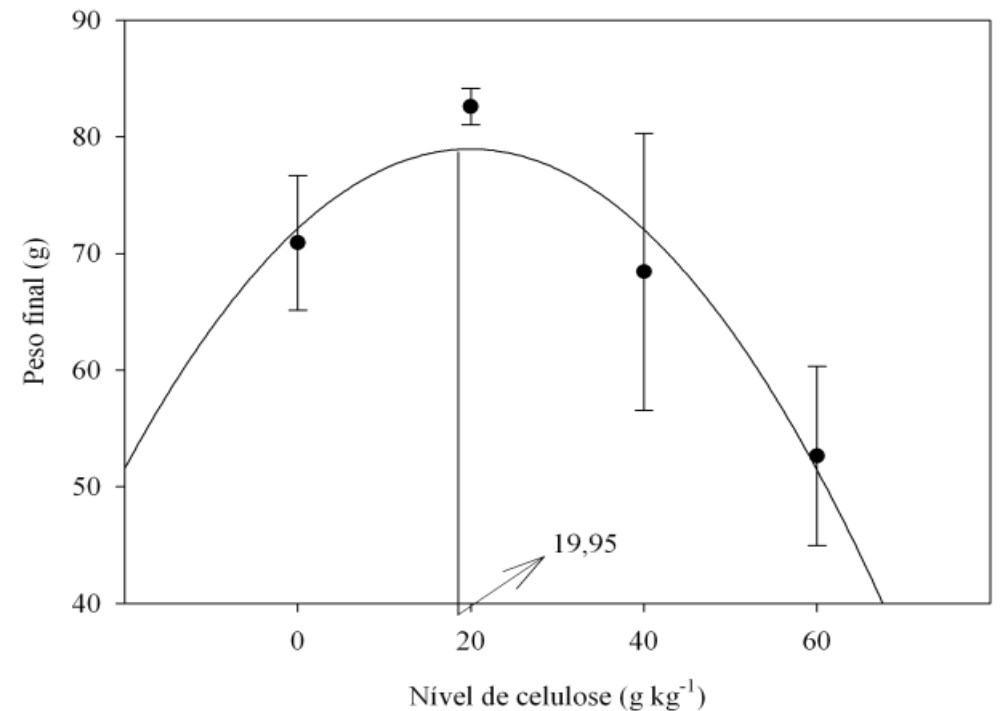

Figura 1. Peso final de juvenis de tambacu de acordo com o nível de inclusão de celulose 
Rev. Bras. Saúde Prod. Anim., Salvador, v.15, n.4, p.947-956 out./dez., 2014 http://www.rbspa.ufba.br ISSN 15199940

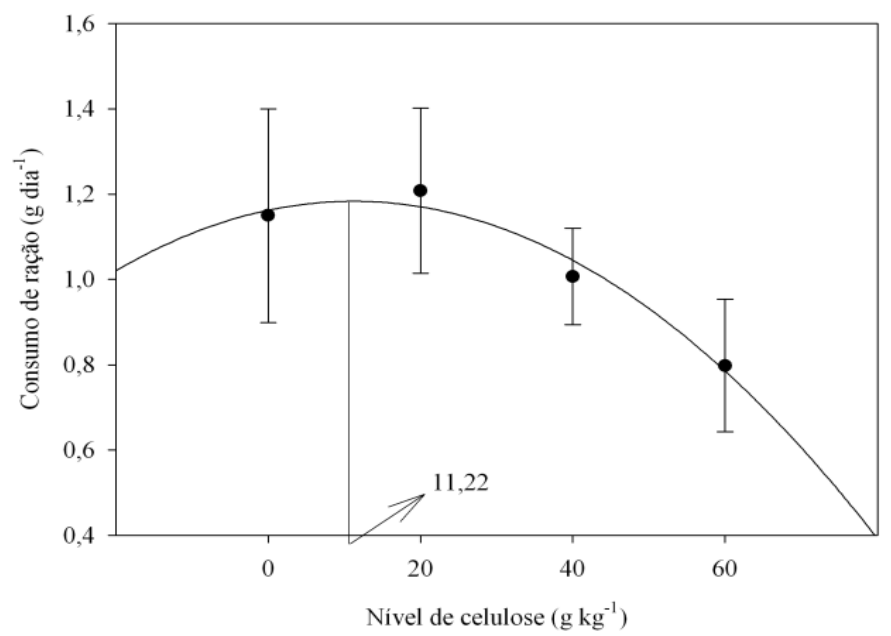

Figura 2. Consumo de ração de juvenis de tambacu de acordo com o nível de inclusão de celulose

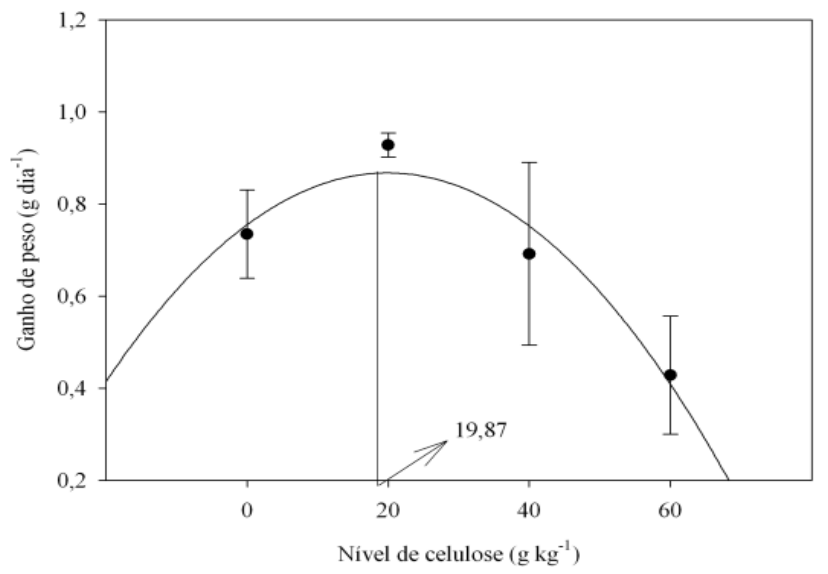

Figura 3. Ganho de peso de juvenis de tambacu de acordo com o nível de inclusão de celulose

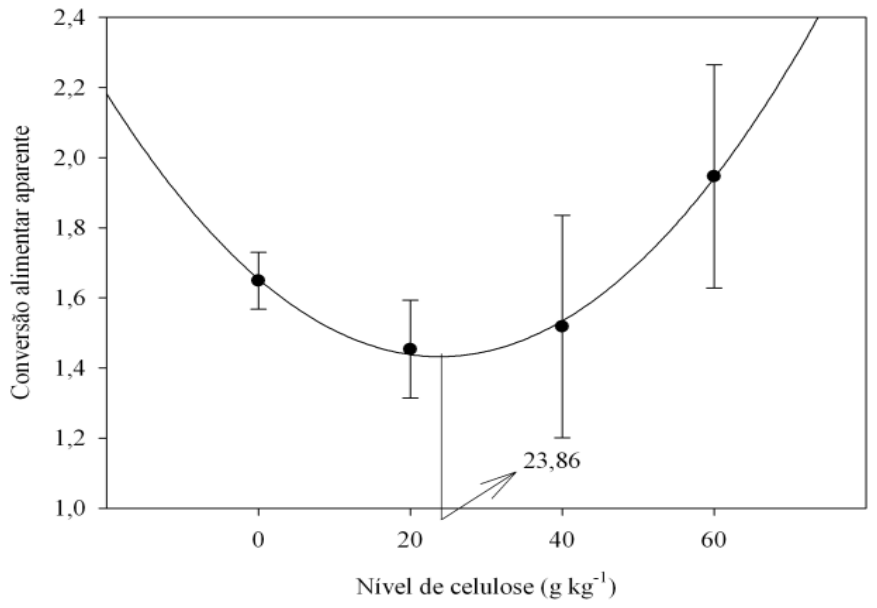

Figura 4. Conversão alimentar aparente de juvenis de tambacu de acordo com o nível de inclusão de celulose 
Contrastando com os resultados obtidos, em experimento com piracanjuba (Brycon orbignyanus) (17,40 $\pm 3,06 \mathrm{~g})$, Garcia et al. (1999) avaliaram níveis crescentes $\left(50 ; 70 ; 80\right.$ e $\left.90 \mathrm{~g} \mathrm{~kg}^{-1}\right)$ de fibra bruta nas rações e observaram maior ganho de peso em peixes alimentados com o nível mais alto avaliado. Por outro lado, Hansen e Storebakken (2007) não observaram redução no desempenho de trutas (Oncorhynchus mykiss) (450g) alimentadas com níveis crescentes de celulose $\left(0 ; 25 ; 50 ; 100 ; 150 \mathrm{~g} \mathrm{~kg}^{-1}\right)$ na ração, Meurer et al. (2003), trabalhando com níveis crescentes de fibra bruta $\left(36,5 ; 47,5 ; 60,0 ; 72,5\right.$ e $\left.85,0 \mathrm{~g} \mathrm{~kg}^{-1}\right)$ para tilápia do nilo $(1,39 \pm 0,03 \mathrm{~g})$, embora tenham observado decréscimo linear da velocidade de trânsito do bolo alimentar com o aumento do nível de fibra bruta, não observaram efeito sobre o desempenho e a sobrevivência e, Lanna et al. (2004b), trabalhando com níveis crescentes de fibra bruta $(60 ; 90 \mathrm{e}$ $120 \mathrm{~g} \mathrm{~kg}^{-1}$ ) para tilápia do nilo $(6,41 \pm 0,05 \mathrm{~g})$, não observaram influência sobre o crescimento.

A molécula de celulose possui três grupos hidroxilas em cada unidade de glicose, o que confere à molécula forte propriedade hidrofílica (BOULOS et al., 2000). No presente estudo, a queda no consumo de ração por juvenis de tambacu alimentados com rações com níveis de celulose a partir de $11,22 \%$, pode ser resultado do aumento no volume do bolo alimentar e, devido a sua elevada capacidade de retenção de água, aumento da sensação de saciedade nos animais (AMIRKOLAIE et al., 2005; HANSEN \& STOREBAKKEN, 2007; RODRIGUES et al., 2010a; THEBAUDIN et al., 1997). Esse fator, aliado à reduzida eficiência na absorção dos nutrientes causada pelo aumento no teor de celulose na ração, uma vez que fibras insolúveis podem reduzir a atividade de enzimas digestivas como amilase, lipase, tripsina e quimiotripsina (SLAVIN, 2005), pode ter sido responsável pelos piores valores de conversão alimentar aparente e, consequentemente, menores peso final $\mathrm{e}$ ganho de peso.

Não houve efeito $(\mathrm{P}>0,05)$ dos níveis de inclusão da celulose sobre a composição química corporal em matéria seca, proteína bruta, extrato etéreo, energia bruta e matéria mineral (Tabela 3).

Tabela 3. Valores médios da composição química corporal de juvenis de tambacu submetidos a diferentes níveis de celulose nas rações

\begin{tabular}{lcccccc}
\hline \multirow{2}{*}{ Variável } & \multicolumn{4}{c}{ Nível de celulose $\left(\mathrm{g} \mathrm{kg}^{-1}\right)$} & \multirow{2}{*}{ Valor de F } & \multirow{2}{*}{ CV $(\%)$} \\
\cline { 2 - 5 } & 0 & 20 & 40 & 60 & & \\
\hline Matéria seca $\left(\mathrm{g} \mathrm{kg}^{-1}\right)$ & 292,3 & 295,0 & 300,5 & 297,0 & 0,32 & 3,12 \\
Proteína bruta $\left(\mathrm{g} \mathrm{kg}^{-1}\right)$ & 622,1 & 610,8 & 617,3 & 628,0 & 1,98 & 4,89 \\
Extrato etéreo $\left(\mathrm{g} \mathrm{kg}^{-1}\right)$ & 251,4 & 279,5 & 259,0 & 278,5 & 2,82 & 6,07 \\
Energia bruta $\left(\mathrm{kcal} \mathrm{kg}^{-1}\right)$ & 4020 & 4177 & 3979 & 4060 & 0,14 & 3,94 \\
Cinzas $\left(\mathrm{g} \mathrm{kg}^{-1}\right)$ & 170,0 & 178,9 & 184,5 & 172,3 & 2,64 & 7,62 \\
\hline
\end{tabular}

Resultados semelhantes foram obtidos por Pedron et al. (2008), que não observaram influência do nível crescente de fibra bruta na ração sobre os parâmetros de composição química do filé de jundiás. Já Lanna et al. (2004b) observaram que a elevação nos teores de fibra bruta em rações para tilápia do nilo reduziram, de forma linear, a matéria seca corporal e 
Rev. Bras. Saúde Prod. Anim., Salvador, v.15, n.4, p.947-956 out./dez., 2014 http://www.rbspa.ufba.br ISSN 15199940

aumentou o teor de proteína bruta corporal sem afetar o extrato etéreo.

Rodrigues et al. (2010b) observaram aumento na proteína bruta e matéria mineral e redução nos valores de extrato etéreo corporais à medida em que aumentaram os níveis de inclusão de fibra bruta em rações para juvenis de pacu. Comparando-se aos resultados obtidos com juvenis de tambacu, mesmo que a fibra tenha a capacidade de reduzir a absorção de gorduras no intestino (SLAVIN, 2005) e, consequentemente, a deposição de gordura corporal, possivelmente, os níveis avaliados no presente estudo não foram suficientes para que houvesse alteração desse parâmetro na composição corporal dos peixes.

As rações com maiores níveis de inclusão da celulose afetaram negativamente o desempenho de juvenis de tambacu, todavia as modificações fisiológicas e metabólicas provocadas por essas rações não foram suficientes para afetar a composição corporal.

\section{AGRADECIMENTOS}

À Fundação de Amparo à Pesquisa do Estado da Bahia (Fapesb - BOL0436/2011), ao Conselho Nacional de Desenvolvimento Cientifico e Tecnológico (CNPq - 482795/20077) e à Coordenação de Aperfeiçoamento de Pessoal de Nivel Superior (Capes - PROCAD 2343/2008) pelo apoio financeiro; 'a Ceplac pela doação dos juvenis de tambacu.

\section{REFERÊNCIAS}

ABIMORAD, E.G.; CARNEIRO, D.J. Métodos de coleta de fezes e determinação dos coeficientes de digestibilidade da fração protéica e da energia de alimentos para o pacu, Piaractus mesopotamicus (Holmberg,

\section{7). Revista Brasileira de}

Zootecnia, v.33, n.5, p.1101-1109, 2004.

AMIRKOLAIE, K.A.; LEENHOUWERS, J.I.; VERRETH, J.A.J.; SCHRAMA, J.W. Type of dietary fiber (soluble versus insoluble) influences digestion, faeces characteristics and faecal waste production in Nile tilapia (Oreochromis niloticus). Aquaculture Research, v.36, n.12, p.1157-1166, 2005.

BOULOS, N.N.; GREENFIELD, H.; WILLS, R.B.H. Water holding capacity of selected soluble and insoluble dietary fiber. International Journal of Food Properties, v.3, n.2, p.217-231, 2000.

CUMMINGS, J.H.; EDMOND, L.M.; MAGEE, E.A. Dietary carbohydrates and health: do we still need the fiber concept? Clinical Nutrition, v.1, p.517, 2004.

FABREGAT, T.E.H.P.; RODRIGUES; L.A.; NASCIMENTO, T.M.T.; URBINATI, E.C.; SAKOMURA, N.K.; FERNANDES, J.B.K. Fontes de fibra na alimentação do pacu: desempenho, composição corporal e morfometria intestinal. Arquivo Brasileiro de Medicina Veterinária e Zootecnia, v.63, n.6, p.1533-1540, 2011.

GARCIA, R.E.; PEZZATO, L.E.; FILHO, E.Z.; VICENTINI, C.A. Utilização da fibra bruta na nutrição de piracanjuba (Brycon orbignyanus). Acta Scientiarum, v.21, n.3, p.725-731, 1999.

HANSEN, J.O.; STOREBAKKEN, T. Effects of dietary cellulose level on pellet and nutrient digestibilities in rainbow trout (Oncorhynchus mykiss). Aquaculture, v.272, n.1-4, p.458-465, 2007. 
Rev. Bras. Saúde Prod. Anim., Salvador, v.15, n.4, p.947-956 out./dez., 2014 http://www.rbspa.ufba.br ISSN 15199940

KROGDAHL, A.; HEMRE, G.I.; MOMMSEN, T.P. Carbohydrates in fish nutrition: digestion and absorption in post larval stages. Aquaculture nutrition, v.11, n.2, p.103-122, 2005.

LANNA, E.A.T.; PEZZATO, L.E.; CECON, P.R.; FURUYA, W.M.; BOMFIM, M.A.D. Digestibilidade aparente e trânsito gastrintestinal em tilápia do Nilo (Oreochromis niloticus), em função da fibra bruta da dieta.

Revista Brasileira de Zootecnia, v.33, n.6, p.2186-2192, 2004a.

LANNA, E.A.T.; PEZZATO, L.E.; FURUYA, W.M.; VICENTINI, C.A.; CECON, P. R.; BARROS, M.M. Fibra bruta e óleo em dietas práticas para alevinos de tilápia do Nilo (Oreochromis niloticus). Revista Brasileira de Zootecnia, v.33, n.6, p.2177-2185, 2004b.

LEENHOUWERS, J.J.; VELD, M.T.; VERRETH, A.J.; SCHRAMA, J.W. Digesta characteristics and performance of African catfish (Clarias gariepinus) fed cereal grains that differ in viscosity. Aquaculture, v.264, n.1-4, p.330-341, 2007.

MEURER, F.; HAYASHI, C.; BOSCOLO, W.R. Fibra bruta para alevinos de tilápia do Nilo (Oreochromis niloticus, L). Revista Brasileira de Zootecnia, v.32, n.2, p.256-261, 2003.

PEDRON, F.A.; NETO, J.R.; EMANUELLI, T.; SILVA, L.P.; LAZZARI, R.; CORRÊIA, V.; BERGAMIN, G.T.; VEIVERBERG, C.A. Cultivo de jundiás alimentados com dietas com casca de soja ou de algodão. Pesquisa Agropecuária Brasileira, v.43, n.1, p.93-98, 2008.
PEREIRA, M.C.; AZEVEDO, R.V.; BRAGA, L.G.T. Óleos vegetais em rações para o híbrido tambacu (macho Piaractus mesopotamicus x fêmea Colossoma macropomum). Revista Brasileira de Saúde e Produção Animal [online], v.12, n.2, p.551-562, 2011.

RODRIGUES, L.A.; FABREGAT, T.E.H.P.; FERNANDES; NASCIMENTO, T.M.T.; SAKOMURA, N.K. Digestibilidade e tempo de trânsito gastrintestinal de dietas contendo níveis crescentes de fibra bruta para pacu. Acta

Scientiarum. Animal Sciences, v.32, n.2, p.169-173, 2010a.

RODRIGUES, L.A.; FERNANDES, J.B.K.; FABREGAT, T.E.H.P.; SAKOMURA, N.K. Desempenho produtivo, composição corporal e parâmetros fisiológicos de pacu alimentado com níveis crescentes de fibra. Pesquisa Agropecuária

Brasileira, v.45, n.8, p.897-902, 2010 b.

R Development Core Team. $R$ : A language and environment for statistical computing. R. Foundation for Statistical Computing, Vienna, Austria. ISBN 3900051-07-0, 2011.URL:

$<$ http://www.R-project.org/>.

RODRIGUES, A.P.O.; GOMINHOROSA, M.D.C.; CARGNINFERREIRA, E.; FRANCISCO, A.; FRACALOSSI, D.M. Different utilization of plant sources by the omnivores jundia catfish (Rhamdia quelen) and Nile tilápia (Oreochromis niloticus). Aquaculture Nutrition, v.18, n.1, p.65-72, 2012.

SILVA, D.J.; QUEIROZ, A.C. Análise de alimentos: métodos químicos e biológicos. 3. ed. Viçosa: Universidade Federal de Viçosa. 2002. 235p. 
Rev. Bras. Saúde Prod. Anim., Salvador, v.15, n.4, p.947-956 out./dez., 2014 http://www.rbspa.ufba.br ISSN 15199940

SLAVIN, J.L. Dietary fiber and body weight. Nutrition, v.21, n.3, p.411-418, 2005 .

THEBAUDIN, J.Y.; LEFEBVRE, A.C.; HARRINGTON, M.; BOURGEOIS, C.M. Dietary fibers: nutritional and technological interest. Trends in Food Science and Technology, v.8, n.2, p.41-48, 1997.

WATANABE, A.L.; VIEGAS, E.M.M.; GONÇALVES, L.U.Levels of yeast and its by-products on pacu juveniles feeding. Revista Brasileira de Zootecnia, v.39, n.3, p.447-453, 2010.

Data de recebimento: 21/02/2014

Data de aprovação: 01/12/2014 